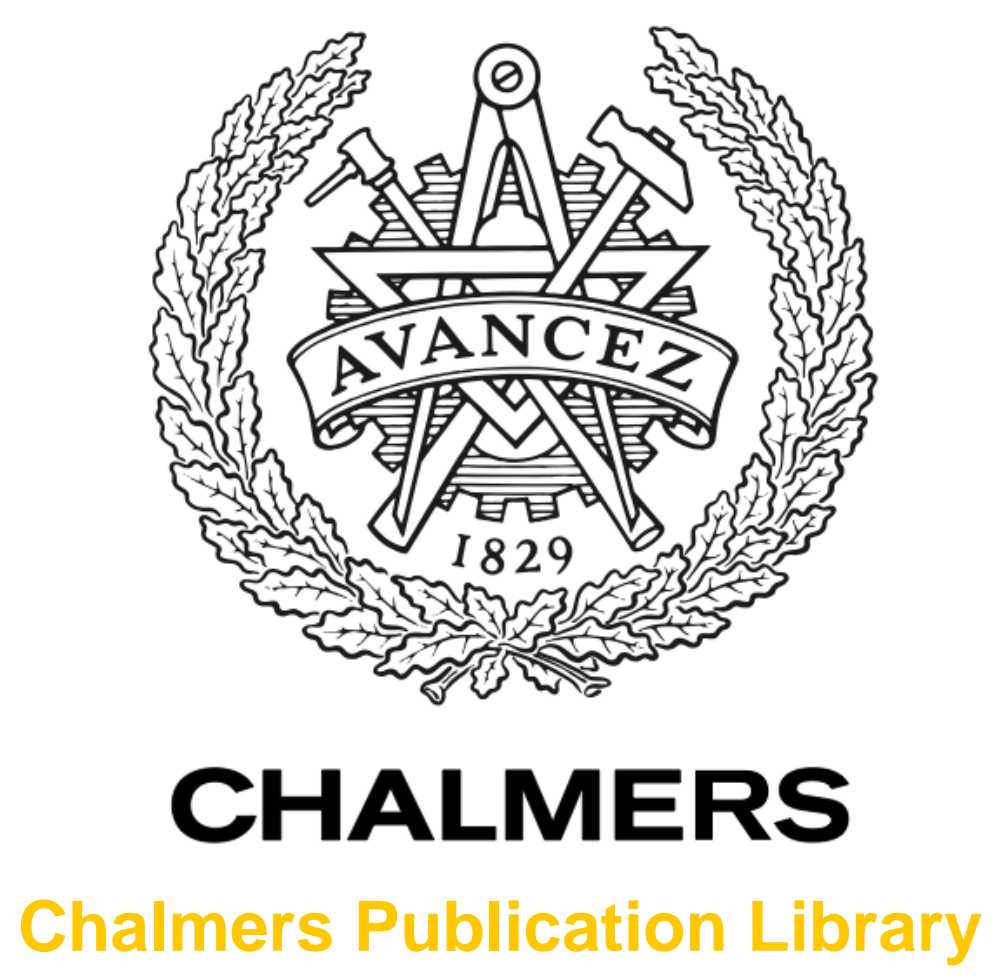

Inverse dispersion engineering in silicon waveguides

This document has been downloaded from Chalmers Publication Library (CPL). It is the author's version of a work that was accepted for publication in:

Journal of the Optical Society of America. B, Optical physics (ISSN: 0740-3224)

Citation for the published paper:

Castello-Lurbe, D. ; Torres Company, V. ; Silvestre, E. (2014) "Inverse dispersion engineering in silicon waveguides". Journal of the Optical Society of America. B, Optical physics, vol. 31(8), pp. 1829-1835.

Downloaded from: http://publications.lib.chalmers.se/publication/201112

Notice: Changes introduced as a result of publishing processes such as copy-editing and formatting may not be reflected in this document. For a definitive version of this work, please refer to the published source. Please note that access to the published version might require a subscription.

Chalmers Publication Library (CPL) offers the possibility of retrieving research publications produced at Chalmers University of Technology. It covers all types of publications: articles, dissertations, licentiate theses, masters theses, conference papers, reports etc. Since 2006 it is the official tool for Chalmers official publication statistics. To ensure that Chalmers research results are disseminated as widely as possible, an Open Access Policy has been adopted.

The CPL service is administrated and maintained by Chalmers Library. 


\title{
Inverse dispersion engineering in silicon waveguides
}

\author{
David Castelló-Lurbe, ${ }^{1, *}$ Victor Torres-Company, ${ }^{2}$ and Enrique Silvestre ${ }^{1}$ \\ ${ }^{1}$ Departament d'Òptica, Universitat de València, 46100 Burjassot, Spain \\ ${ }^{2}$ Department of Microtechnology and Nanoscience (MC2), Chalmers University of Technology, \\ 41296 Gothenburg, Sweden \\ ${ }^{*}$ Corresponding author: david.castello-lurbe@uv.es
}

Received March 10, 2014; revised May 22, 2014; accepted June 13, 2014; posted June 19, 2014 (Doc. ID 207805); published July 10, 2014

\begin{abstract}
We present a numerical tool that searches an optimal cross section geometry of silicon-on-insulator waveguides given a target dispersion profile. The approach is a gradient-based multidimensional method whose efficiency resides on the simultaneous calculation of the propagation constant derivatives with respect to all geometrical parameters of the structure by using the waveguide mode distribution. The algorithm is compatible with regular mode solvers. As an illustrative example, using a silicon slot hybrid waveguide with 4 independent degrees of freedom, our approach finds ultra-flattened (either normal or anomalous) dispersion over $350 \mathrm{~nm}$ bandwidth in less than 10 iterations. (c) 2014 Optical Society of America

OCIS codes: $\quad$ (130.2035) Dispersion compensation devices; (130.3120) Integrated optics devices; (130.4310)

Nonlinear.

http://dx.doi.org/10.1364/JOSAB.31.001829
\end{abstract}

\section{INTRODUCTION}

Chromatic dispersion is one of the most important properties that controls the physical behavior of waveguides in both the linear and nonlinear regimes. Its design is key to achieving high performance in applications such as dispersion compensation [1] , parametric amplification [2] , wavelength conversion $[3,4]$, and supercontinuum generation $[5,6]$. The waveguide dispersion not only depends on its constituent materials, but also displays a high sensitivity to changes in geometry (see e.g., [7]). This is especially true for silicon-on-insulator (SOI) waveguides $[2-6,6]$. Here, there is a high-index-contrast between materials and the possibility exists to engineer the design at the nanometer scale. These characteristics enable an unprecedented control on the waveguide's dispersion properties.

Algorithms allowing for the optimization of the design are tools of great interest. The most widespread techniques rely on commercially available software that calculates the dispersion profile given a certain structure. This favors direct optimization methods by trial and error. For example, the zero dispersion can be tuned within the $C$-band by adjusting the height and width of a strip waveguide $[\underline{4}, \underline{5}, \underline{9}]$. Notwithstanding, there are advanced configurations with multiple geometrical degrees of freedom that allow for a much finer control of the dispersion curve [10-12]. The multidimensional optimization renders impractical the application of the direct methods.

Gradient-based algorithms (GBAs) [13] speed up the convergence process with estimations of the direction in the parameter space (e.g., the cross section geometries of the waveguide) that lead to the largest improvement of the magnitude to be optimized (e.g., dispersion). Topology optimization is a particular GBA that defines some feature (e.g., the refractive index) of all the sampling points of the cross section as free parameters. Hence, it can manage a great number of degrees of freedom [14]. It has been previously used in nanophotonics design [14] and dispersion-compensating fibers [15].
In [16], our group proposed an inverse dispersion engineering approach, based on a multidimensional gradient algorithm, to calculate directly the derivatives of the propagation constant with respect to all the structural parameters of a waveguide in a full vectorial framework. It is worth emphasizing that this result does not rely on any particular technique for solving the wave equation. Certainly, this approach circumvents one of the main disadvantages of direct methods and, therefore, provides results in just a few iterations. Previously, it was successfully applied in the context of photonic crystal fibers $[16,17]$.

In this work, we apply this technique to SOI waveguides. Due to its intrinsic high index contrast, we take special care of the vectorial nature of the problem, since the axial component in electric and magnetic fields can be significant in these waveguides [18]. In the next section, we explain our GBA, paying particular attention to the specific numerical issues related to high-index-contrast waveguides. Section 3 is devoted to the numerical results computed through our procedure, including optimizations under additional constraints, and in Section 4 we deal with an analysis of the solutions achieved. Finally, the main conclusions are drawn in Section $\underline{5}$.

\section{GRADIENT-BASED OPTIMIZATION ALGORITHM}

GBAs are the first choice in multidimensional optimization when the derivatives of the fitness function are available [13]. Therefore, with the aim of tackling the dispersion engineering of waveguides, a procedure based in the evaluation of the derivatives of the propagation constant $\beta$ with respect to the design parameters was proposed in [16]. In this section, we present a close expression for those derivatives well-functioning, even in the case of high-index-contrast waveguides, and include a detailed description of their implementation. 
For dispersion engineering purposes, a simple definition for the merit function in the optimization procedure is the mean squared of the difference between the group-velocity dispersion (GVD), namely $\beta_{2}(\omega)=\mathrm{d}^{2} \beta / \mathrm{d} \omega^{2}$, of a particular structure represented by $p$ and a target GVD:

$$
\chi^{2}(p)=\frac{1}{N_{\omega}} \sum_{k=1}^{N_{\omega}}\left(\beta_{2}\left(p ; \omega_{k}\right)-\beta_{2}^{\text {target }}\left(\omega_{k}\right)\right)^{2},
$$

where $p=\left(p_{1}, \ldots, p_{N}\right)$ is the set of parameters defining the waveguide degrees of freedom. This expression corresponds to the variance of the dispersion with respect to the target dispersion profile in the frequency range of design, and the optimum configuration will correspond to the minimum of $\chi^{2}$.

To reach that minimum, we follow a sequential linear programming (SLP) strategy for producing a series of quadratic problems to be minimized. To be more precise, given a point in the parameter space $p_{(m)}$, we can obtain the linear approximation of $\beta_{2}(p)$ around it:

$$
\beta_{2}^{\operatorname{lin}}(p ; \omega)=\beta_{2}\left(p_{(m)} ; \omega\right)+\partial_{p} \beta_{2}\left(p_{(m)} ; \omega\right) \cdot\left(p-p_{(m)}\right)
$$

and, accordingly, a local (quadratic) approximation of the actual merit function:

$$
\chi_{\mathrm{loc}}^{2}(p)=\frac{1}{N_{\omega}} \sum_{k=1}^{N_{\omega}}\left(\beta_{2}^{\operatorname{lin}}\left(p ; \omega_{k}\right)-\beta_{2}^{\text {target }}\left(\omega_{k}\right)\right)^{2} .
$$

Thus, if $\partial_{p} \beta_{2}$ is known, $\chi_{\text {loc }}^{2}$ can be built, and its minimum $p_{(m+1)}$, can be easily determined with no additional computational effort. This new point is expected to be closer to the target and can be used as the starting point in a new iteration of the procedure.

In Appendix A, we go one step further than [16] and derive the following expression for the gradient of the propagation constant in the parameter space:

$$
\partial_{p} \beta=\frac{\epsilon_{0} \omega}{2} \frac{\int_{S}\left(-\mathbf{e}_{\mathrm{t}} \cdot\left(\partial_{p} \epsilon_{\mathrm{tt}}\right) \mathbf{e}_{\mathrm{t}}+\left(\partial_{p} \epsilon_{z z}\right) e_{z}^{2}\right) \mathrm{d} S}{\int_{S}\left(\mathbf{e}_{\mathrm{t}} \times \mathbf{h}_{\mathrm{t}}\right) \cdot \hat{\mathbf{z}} \mathrm{d} S},
$$

where integrals extend to the entire transverse domain where fields are defined $S$, and involve components of the electromagnetic field and derivatives of the dielectric tensor. The subscript $t$ indicates transverse components, $\epsilon_{0}$ is the permittivity of the vacuum, and $\omega$ is the light frequency. It is worth emphasizing a couple of points around Eq. (4). On the one hand, $\left(\partial_{p} \epsilon_{z z}\right) e_{z}^{2}$ is the explicit contribution of the vector nature of the electromagnetic field and must be taken into account for high-index-contrast waveguides [18]. On the other hand, Eq. (4) enables the calculation of $\partial_{p} \bar{\beta}$ by means of the mode fields of the waveguide at only one parameter configuration. This means that there is no need to know the propagation constant at any other point $p+\delta p$ of the multidimensional parameter space to compute its derivatives.

If we focus on geometrical parameters defining waveguides composed of homogeneous materials, then a smoothed effective dielectric tensor $\hat{\epsilon}$ should be used [19]. This description of the material distribution allows us to evaluate these derivatives in an easy way, avoiding infinities at the discontinuities, whereas derivatives are nonzero only near interfaces. This tensor $\hat{\epsilon}$ must be defined at each spatial sampling point, according to the effective-medium theory [20], as explained in Appendix B.

\section{A. Accurate Calculations for High-Index-Contrast Waveguides}

From the numerical point of view, and taking into account the discretization of the integration domain $S$, the integral in the numerator of Eq. (4) can present a considerable error in its evaluation owing to the strong discontinuity around the interface of the normal component of the electric field. That difficulty can be overcome if we choose a local basis for the electric field defined by the unit vectors $(\hat{\mathbf{n}}, \hat{\mathbf{T}}, \hat{\mathbf{z}})$ : the normal and the tangent to the interface in the transverse plane, $\hat{\mathbf{n}}$ and $\hat{\mathbf{T}}$, and the propagation direction $\hat{\mathbf{z}}$. On this basis, Eq. ( $\underline{4}$ ) can be rewritten as

$$
\partial_{p} \beta=\frac{\epsilon_{0} \omega}{2} \frac{\int_{S}\left(\left(\partial_{p} \epsilon_{n n}^{-1}\right) d_{n}^{2}-\left(\partial_{p} \epsilon_{T T}\right) e_{T}^{2}+\left(\partial_{p} \epsilon_{z z}\right) e_{z}^{2}\right) \mathrm{d} S}{\int_{S}\left(\mathbf{e}_{\mathrm{t}} \times \mathbf{h}_{\mathrm{t}}\right) \cdot \hat{\mathbf{z}} \mathrm{d} S},
$$

where $d_{n}$ is the component of the electric displacement field normal to the interface and $e_{T}$ is the component of the electric field tangent to the interface in the transverse plane. It is worth remembering that the dielectric tensor is diagonal in the new local basis since the effective medium around the interface corresponds to a uniaxial anisotropic medium [19], and its extraordinary axis is normal to the interface (parallel to $\hat{\mathbf{n}}$ ).

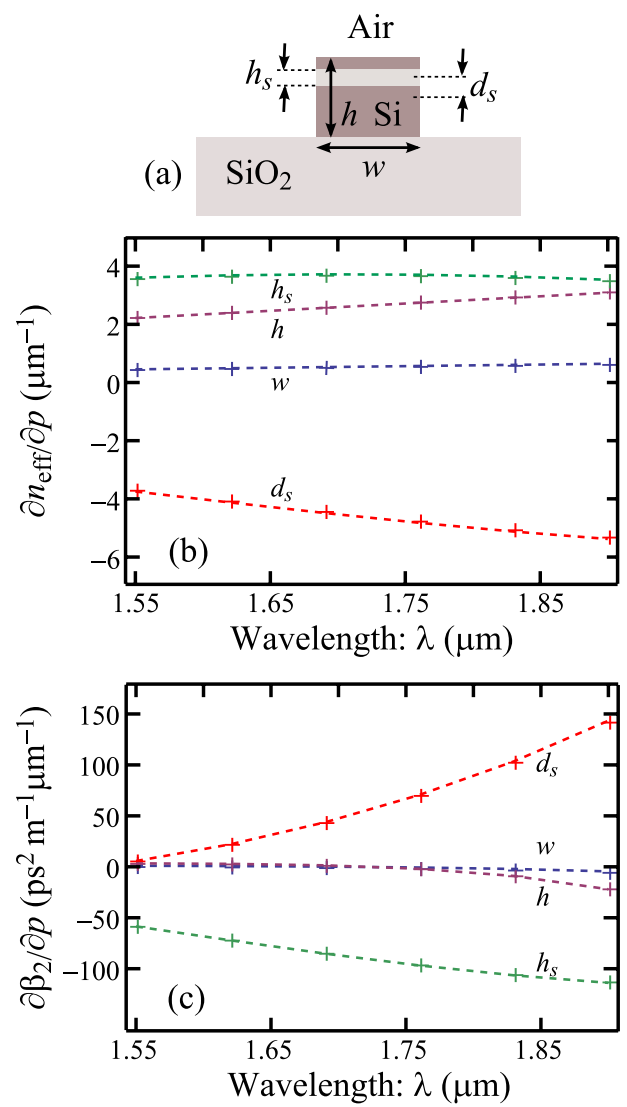

Fig. 1. (a) Outline of the slot waveguide under study [11]. Comparison of the derivatives of the effective refractive index (b) and the group-velocity dispersion parameter (c) with respect to the geometrical parameters $h, w, h_{s}$, and $d_{s}$, shown in (a), calculated numerically (dashed curves) and by Eq. (5) (crosses). 
Therefore, Eq. (5) extends the applicability of Eq. (4) to high-index-contrast waveguides.

To check the numerical precision of Eq. (5), we consider a silicon strip waveguide with a horizontal silica slot, proposed in [11] by Zhang et al., which is shown in Fig. 1(a). These waveguides present four geometrical degrees of freedom, which we define as the width of the waveguide $(w)$, the height of the waveguide $(h)$, the height of the slot $\left(h_{s}\right)$, and the position of the slot center with respect to the waveguide center $\left(d_{s}\right)$. This is a computationally demanding structure owing to the narrow low-index layer in the middle of a high-index, strongly confining waveguide, near the maximum of field density. Indeed, the light-guiding mechanism of the transverse magnetic- (TM)-mode (vertically polarized) of this kind of waveguide induces a strong change on the normal component of the electric field at the slot lower interface [11]. Let us consider the following arbitrary configuration: $w=650 \mathrm{~nm}$, $h=460 \mathrm{~nm}, h_{s}=50 \mathrm{~nm}$, and $d_{s}=115 \mathrm{~nm}$. We calculate the propagation constant and the fields of the TM-mode using an iterative two-dimensional procedure [21] within a squared sampling window of $1.8 \mu \mathrm{m}$ long, and a sampling distance of $6.25 \mathrm{~nm}$. Furthermore, if a sampling grid parallel to the structure were used, then the derivatives would be highly sensitive to the position of the sampling points around the slot horizontal interfaces. To deal with this numerical problem, we rotate the structure slightly $\left(1.5^{\circ}\right)$ with respect to the sampling grid. In Fig. 1(b), we compare results from Eq. (5) with those computed numerically (in particular, solving the wave equation also for neighbor configurations by changing the initial value of each parameter by $\pm 0.5 \mathrm{~nm}$ and $\pm 1.0 \mathrm{~nm}$, fitting the results to a second order polynomial, and calculating its derivative at the initial point). We can observe that the analytical derivatives are in very good agreement with the numerical calculations.

Equation (5) allows us to easily compute the first derivatives of $\beta$ and engineer magnitudes that depend algebraically on $\beta$. Nevertheless, there is no closed expression for calculating higher-order derivatives in a straightforward manner [21]. Despite this, if we are interested in optimizing the chromatic dependency of a derivative of $\beta$, then we just need to evaluate Eq. (5) for different frequencies, fit those data as a function of $\omega$, and perform successive derivatives with respect to frequency. This approach is fast and, as can be appreciated in Fig. 1(c), keeps a high accuracy.

\section{NUMERICAL RESULTS}

We illustrate our approach in Fig. 2, where we plot five different examples of the optimization processes with different
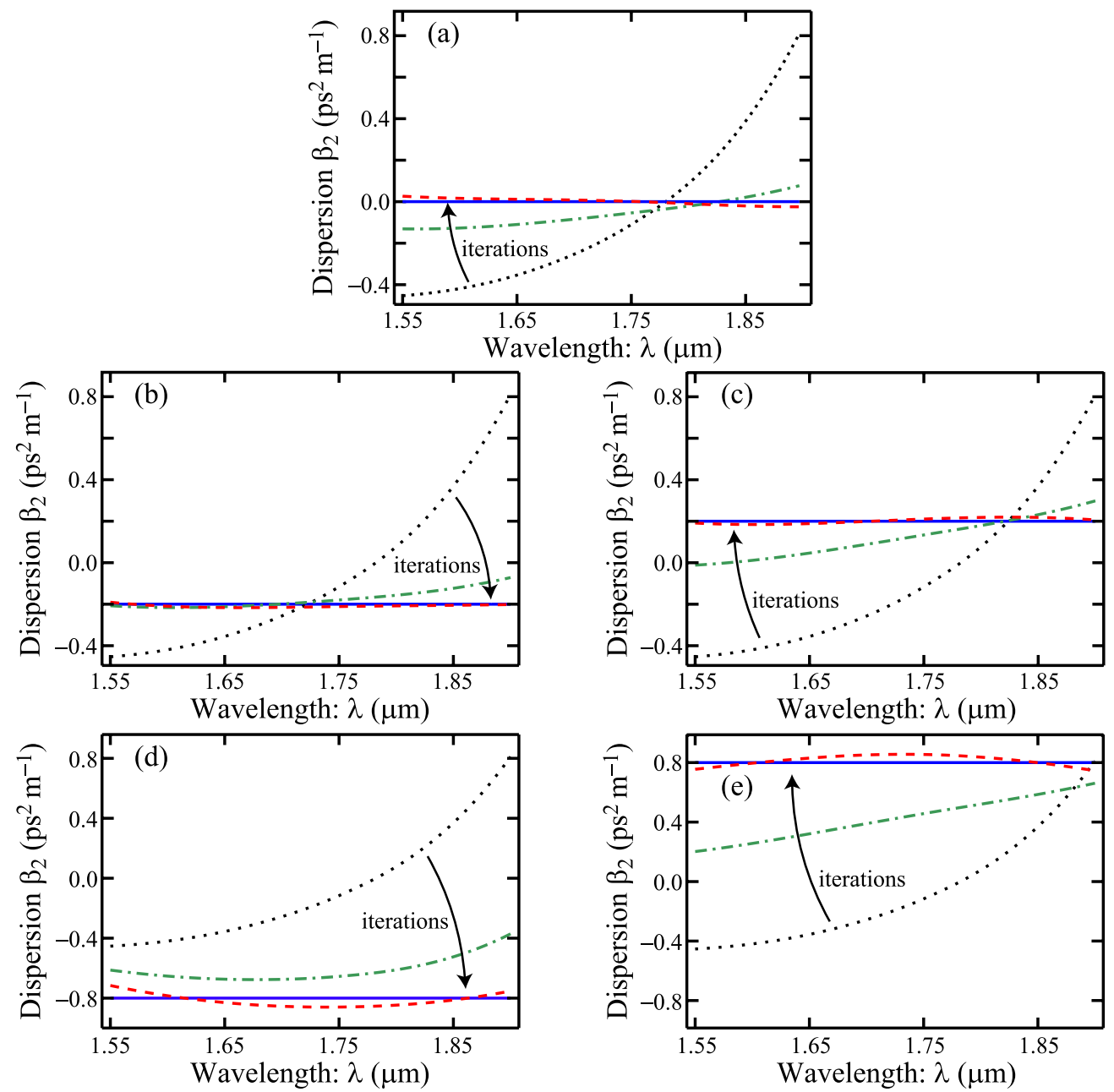

Fig. 2. Five examples of optimization starting from the same geometry (dotted black curve, see details in the text) and with five different flattened dispersion profiles as a goal (solid blue line); namely, zero (a), low and anomalous (b), low and normal (c), high and anomalous (d), and high and normal (e). Dispersion profiles between the first iteration (dotted-dashed green curve) and the last one (dashed red line) are omitted. 
flattened dispersion profiles over $350 \mathrm{~nm}$, in the range $1.55 \mu \mathrm{m} \leq \lambda \leq 1.90 \mu \mathrm{m}$ as target: namely, $\beta_{2}(\omega)=0, \pm 0.2$ and $\pm 0.8 \mathrm{ps}^{2} \mathrm{~m}^{-1}$. For all five cases, we start from the same waveguide configuration [11], hence, showing this choice is not particularly relevant. This corresponds to the geometry used to test the accuracy of the analytical derivatives in the previous section, whose dispersion is far from being flattened. In our simulations, we describe the refractive index of silicon and silica using the Sellmeier coefficients provided in [22]. In these examples, six wavelengths in the above range are considered as the points used for evaluating the local approximation of the merit function [Eq. (3)].

It is worth noting that the target curves are recovered to different extents. In other words, the achievable minimum of $\chi^{2}$ depends on the target curve and on the waveguide's degrees of freedom. To ensure that the procedure converges properly, it is convenient to moderate the speed of convergence. In Fig. 3, the evolution of the parameters at each step along the procedure is shown, in which we have allowed variations up to $10 \%$ in each step and we consider the process has converged when the difference of $\chi$ between two successive steps is smaller than $0.001 \mathrm{ps}^{2} \mathrm{~m}^{-1}$. The specific designs obtained after convergence are indicated in Table 1 . Note that, for the $\beta_{2}=0$ case, the slot parameters, which are the most sensitive ones [see Fig. 1(c)], are in close agreement with those reported in [12], where $h_{s}=40 \mathrm{~nm}$ and $d_{s}=104 \mathrm{~nm}$. We emphasize that curves close to the target are found in a few steps (see Fig. 3). This illustrates the efficiency of this approach compared with trial-and-error methods.

Since Eq. (1) has been defined in this work for engineering $\beta_{2}$, other magnitudes can be altered during the optimization process. In the cases shown in Fig. 2, for instance, the effective refractive index grows as $\beta_{2}$ approaches its target and, therefore, modes become more confined. The effective index behavior is associated with the positive sign of the derivatives with respect to the height of the waveguide, and the fact that this parameter has been increased along the processes [see Figs. 1(a) and 3].

\section{A. Inclusion of Feasibility Restrictions}

The manufacturing of integrated waveguides is, of course, constrained by the limitations of the fabrication method. Therefore, any realistic design procedure must include this kind of additional requirement. Our algorithm also allows us to deal with these fabrication restrictions. They are implemented as constraints in the variables of the local merit function $\chi_{\text {loc }}$, i.e., limiting the possible values of $p$ or bounding them.
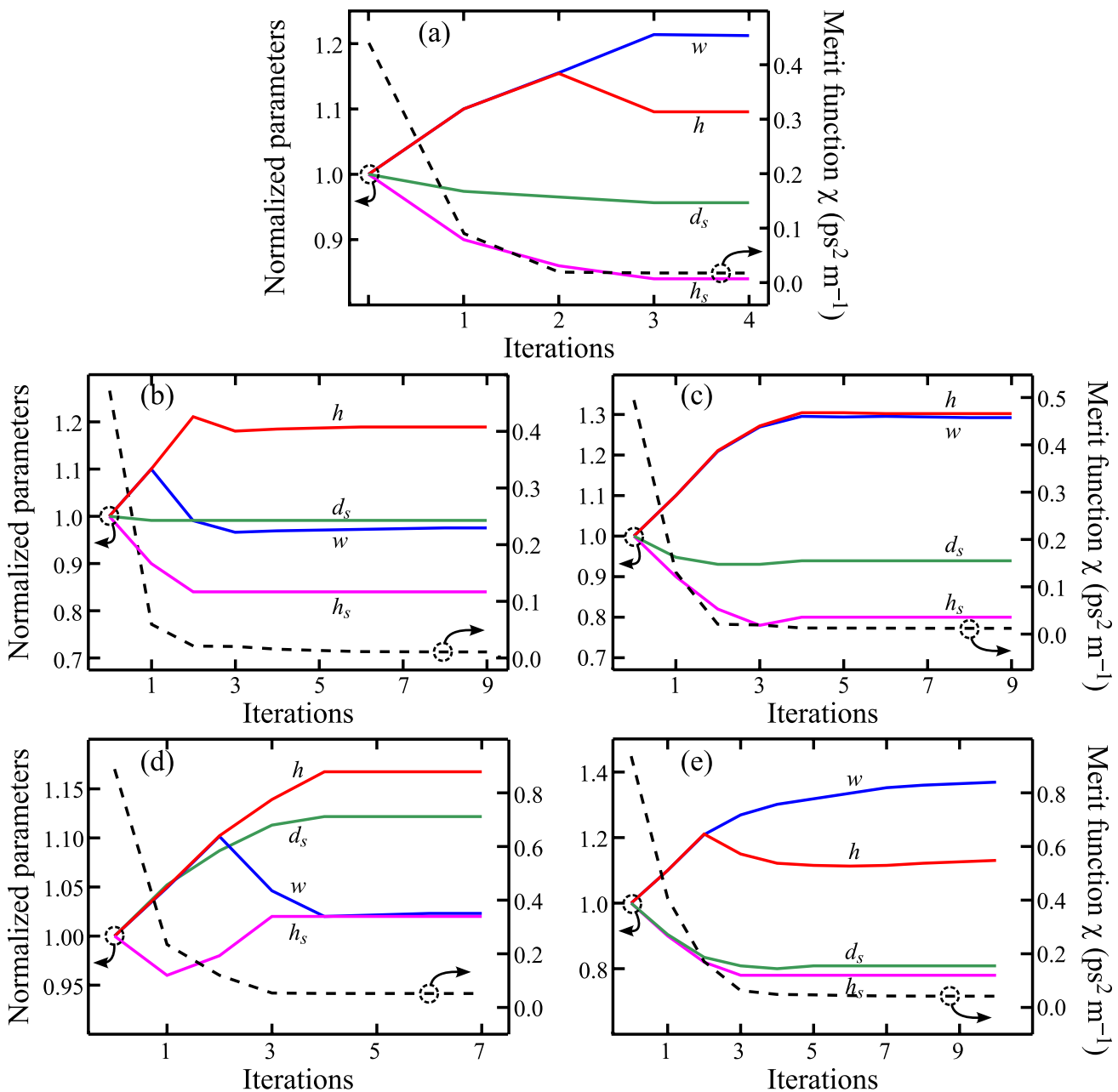

Fig. 3. Plot of the evolution of the geometrical parameters during the optimization procedure of the same five examples shown in Fig. 2 , normalized to its starting values (solid colored curves, left-hand axis). Dashed black curves represent the evolution of the square root of the merit function, the standard deviation of $\beta_{2}$ with respect to the target in the design frequency range (right-hand axis). 
Table 1. Geometrical Parameters and Merit Function after Convergence for the Five Dispersion Targets (Fig. 2)

\begin{tabular}{lcccccc}
\hline$\beta_{2}^{\text {target }}\left(\mathrm{ps}^{2} \mathrm{~m}^{-1}\right)$ & iter. & $\chi\left(\mathrm{ps}^{2} \mathrm{~m}^{-1}\right)$ & $w(\mathrm{~nm})$ & $h(\mathrm{~nm})$ & $h_{s}(\mathrm{~nm})$ & $d_{s}(\mathrm{~nm})$ \\
\hline (a) +0.0 & 4 & 0.018 & 788 & 504 & 42 & 110 \\
(b) -0.2 & 8 & 0.011 & 634 & 547 & 42 & 114 \\
(c) +0.2 & 8 & 0.012 & 840 & 599 & 40 & 108 \\
(d) -0.8 & 6 & 0.052 & 665 & 537 & 51 & 129 \\
(e) +0.8 & 9 & 0.042 & 887 & 518 & 39 \\
\hline
\end{tabular}

The dispersion profile is more sensitive to those parameters related to the slot [see Fig. 1(c)]. Therefore, to illustrate the possibility of including some constraints in our algorithm, we have imposed a lower boundary of $45 \mathrm{~nm}$ to the slot height in the optimization processes of Fig. 2 . The dispersion profiles obtained under such a condition are shown in Fig. 4 . Of course, the impact of this constraint depends on each case. In our examples, it affects the two cases of normal dispersion to a greater extent (see results in Fig. 4) since such cases show the narrowest slots in the unrestricted optimization (see Table 1).

\section{ANALYSIS OF TOLERANCES}

In this work, we have engineered the dispersion profile of SOI waveguides in an efficient way, implementing a powerful differential tool that allows us to evaluate, with no additional computational effort, the gradient of the propagation constant in the parameter space. We used a kind of slot waveguide proposed by Zhang et al. [11,12] to illustrate our inverse dispersion engineering proposal. Nonetheless, we cannot omit the practical interest of the specific structures obtained applying our tool. For example, the cross section designs that provide the dispersion curves of Figs. 2(a)-2(c) could have applications for soliton formation in optical microresonators [23] or coherent ultraflat supercontinuum generation [12,17], whereas the dispersion profiles shown in Figs. 2(d) and 2(e) could be used for dispersion compensation of ultrashort pulses [1]. In this sense, a crucial point to test the feasibility of any design is to know how it performs facing inevitable changes with respect to the optimized structure. To this end, and for the sake of completeness, we characterize in this section the sensitivity of the waveguides shown in Fig. 3 to fabrication tolerances. In addition, note that our approach also allows us to analytically evaluate the uncertainty of the effective index or the dispersion due to the fabrication

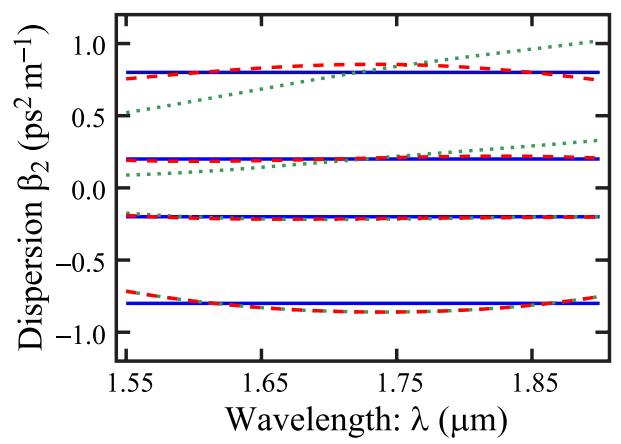

Fig. 4. Last four target (solid blue lines) and optimized (dashed red curves) dispersion profiles shown in Fig. 2 together with those obtained under the restriction $h_{s} \geq 45 \mathrm{~nm}$ (dotted green curves). tolerances by using the standard technique of propagation of errors (see e.g., [16]).

In Fig. 5, the effect of small perturbations of the geometrical parameters on dispersion curves is represented. We take the geometry obtained by means of the optimization process shown in Fig. 2(b) as reference. Since current integrated waveguides can be fabricated with typical tolerances of a few nanometers [24], we have increased and decreased by $2 \mathrm{~nm}$ the parameters of that structure. As expected from Fig. 1(c), the GVD is more sensitive to changes that affect the slot compared with perturbations of the strip.

\section{SUMMARY}

The physical properties of subwavelength waveguides are highly dependent on its geometry due to their small dimensions and high index contrasts. This feature becomes an advantage as long as their cross section can be suitably designed. Usually, optimum waveguide designs are found by varying separately the geometrical degrees of freedom in their transverse planes. Consequently, this trial-and-error procedure becomes much more challenging when one considers advanced designs containing multiple degrees of freedom.

In this work, we overcome this issue by means of an inverse dispersion engineering approach. Given a target for the dispersion curve, we can simultaneously modify all geometrical parameters of the waveguide toward new values where the structure shows dispersion profiles closer to the target. In this way, in a few iterations we find specific structures with dispersion curves matching, as much as possible, the target. The key fact is the possibility of computing the gradient of the propagation constant with respect to all the geometrical parameters by means of the information provided by the analysis of only one geometry. In addition, our algorithm is compatible with any wave equation solver, since it only needs the information of the mode fields.

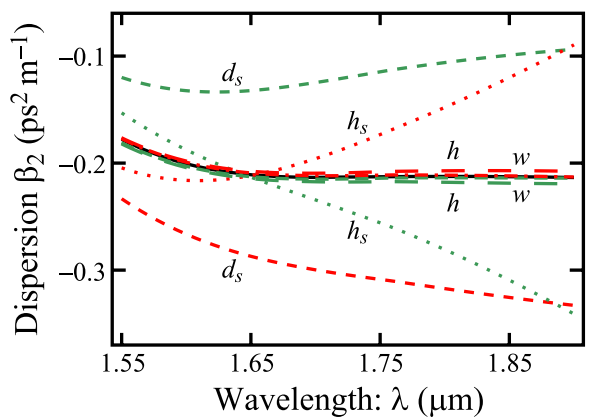

Fig. 5. Plot of the dispersion profiles corresponding to the optimized geometry for the system described in Fig. 2(b) (solid black curve, see details in the text); and the same geometry with each one of the design parameters $\left(h, w, h_{s}\right.$, and $\left.d_{s}\right)$ increased (red lines) or decreased (green lines) by $2 \mathrm{~nm}$. 


\section{APPENDIX A: DERIVATIVE OF THE PROPAGATION CONSTANT}

Let us write the two-dimensional wave equation for the transverse components of the magnetic field of a mode in a waveguide [16],

$$
L \mathbf{h}_{\mathrm{t}}=\left\{\nabla_{\mathrm{t}} \nabla_{\mathrm{t}}^{\mathrm{T}}+\eta \epsilon_{\mathrm{tt}} \eta^{\mathrm{T}} \frac{\omega^{2}}{c^{2}}-\eta \epsilon_{\mathrm{tt}} \nabla_{\mathrm{t}}\left[\epsilon_{z z}^{-1} \nabla_{\mathrm{t}}^{\mathrm{T}} \eta\right]\right\} \mathbf{h}_{\mathrm{t}}=\beta^{2} \mathbf{h}_{\mathrm{t}},
$$

where the subscript $\mathrm{t}$ indicates transverse components, the superscript $\mathrm{T}$ refers to the transpose of a matrix or vector, $\epsilon$ is the effective dielectric tensor [19], and $\eta$ represents the completely antisymmetric tensor in two dimensions $[(0,+1)$, $(-1,0)]$. The above equation can be recognized as an eigenproblem and, therefore, the Hellmann-Feynman theorem [25] could be used for calculating derivatives of its eigenvalues. This strategy has been applied in solving electromagnetic problems in cavities, where eigenvalues are the resonant frequencies $[\underline{14}, \underline{26}]$. However, as the operator $L$, defined by the left-hand side of Eq. (A.1), is nonself-adjoint ( $c f$. [15], where polarization effects are discarded, and $L$ becomes self-adjoint), a generalization based on biorthogonality [27] must be used. The general expression for this mathematical problem turns out to be [16]

$$
\frac{\partial\left(\beta^{2}\right)}{\partial p}=\frac{\left\langle\tilde{\mathbf{h}}_{\mathrm{t}} \mid\left(\partial_{p} L\right) \mathbf{h}_{\mathrm{t}}\right\rangle}{\left\langle\tilde{\mathbf{h}}_{\mathrm{t}} \mid \mathbf{h}_{\mathrm{t}}\right\rangle},
$$

where $p=\left(p_{1}, \ldots, p_{N}\right)$ are the parameters defining the waveguide, $\tilde{\mathbf{h}}_{\mathrm{t}}$ is the eigenvector of the adjoint operator of $L$, whose eigenvalue is the complex conjugate of $\beta^{2}$ [i.e., $L^{\dagger} \tilde{\mathbf{h}}_{\mathrm{t}}=\left(\beta^{2}\right) * \tilde{\mathbf{h}}_{\mathrm{t}}$ ] and, therefore, $\tilde{\mathbf{h}}_{\mathrm{t}}=\eta \mathbf{e}_{\mathrm{t}}^{*}$, being $\mathbf{e}_{\mathrm{t}}$ the transverse components of the electric field of the considered waveguide mode [27]. $\langle\circ \mid \circ\rangle$ stands for the standard scalar product for complex vector functions. It is worth recalling that only the elements explicitly dependent on any $p_{k}$ contribute to the derivation of $L$.

To derive Eq. (4) from Eq. (A.2), first we explicitly write the numerator on the right-hand side in Eq. (A.2),

$$
\begin{aligned}
\left\langle\tilde{\mathbf{h}}_{\mathrm{t}} \mid\left(\partial_{p} L\right) \mathbf{h}_{\mathrm{t}}\right\rangle= & \int_{S} \mathrm{~d} S\left(\frac{\omega^{2}}{c^{2}} \mathbf{e}_{\mathrm{t}}^{\mathrm{T}}\left(\partial_{p} \epsilon_{\mathrm{tt}}\right) \eta^{\mathrm{T}} \mathbf{h}_{\mathrm{t}}\right. \\
& \left.-\mathbf{e}_{\mathrm{t}}^{\mathrm{T}}\left(\partial_{p} \epsilon_{\mathrm{tt}}\right) \nabla_{\mathrm{t}}\left[\epsilon_{z z}^{-1} \nabla_{\mathrm{t}}^{\mathrm{T}} \eta \mathbf{h}_{\mathrm{t}}\right]-\mathbf{e}_{\mathrm{t}}^{\mathrm{T}} \epsilon_{\mathrm{tt}} \nabla_{\mathrm{t}}\left[\left(\partial_{p} \epsilon_{z z}^{-1}\right) \nabla_{\mathrm{t}}^{\mathrm{T}} \eta \mathbf{h}_{\mathrm{t}}\right]\right) .
\end{aligned}
$$

Let us consider now the following Maxwell equations:

$$
\begin{gathered}
\nabla \times \mathbf{H}=-i \sqrt{\frac{\epsilon_{0}}{\mu_{0}}} \frac{\omega}{c} \epsilon \mathbf{E}, \\
\nabla \times \mathbf{E}=i \sqrt{\frac{\mu_{0}}{\epsilon_{0}}} \frac{\omega}{c} \mathbf{H}, \\
\nabla \cdot \mathbf{D}=0 .
\end{gathered}
$$

If we separate the transverse and longitudinal field components, then $\mathbf{E}=\left(\mathbf{e}_{\mathrm{t}}+e_{z} \hat{\mathbf{z}}\right) \exp (i \beta z)$ and $\mathbf{H}=\left(\mathbf{h}_{\mathrm{t}}+h_{z} \hat{\mathbf{z}}\right)$ $\exp (i \beta z)$, and we obtain

$$
\nabla_{\mathrm{t}}^{\mathrm{T}} \eta \mathbf{h}_{\mathrm{t}}=-i \sqrt{\frac{\epsilon_{0}}{\mu_{0}}} \frac{\omega}{c} \epsilon_{z z} e_{z},
$$

$$
\begin{gathered}
\nabla_{\mathrm{t}} e_{z}=-i \sqrt{\frac{\mu_{0}}{\epsilon_{0}}} \frac{\omega}{c} \eta \mathbf{h}_{\mathrm{t}}+i \beta \mathbf{e}_{\mathrm{t}}, \\
\nabla_{\mathrm{t}}^{\mathrm{T}}\left(\epsilon_{\mathrm{tt}} \mathbf{e}_{\mathrm{t}}\right)=-i \beta \epsilon_{z z} e_{z},
\end{gathered}
$$

from Eqs. (A.4), (A.5), and (A.6), respectively. If we take into account Eqs. (A.7) and (A.8), then we can simplify the second term on the right-hand side in Eq. (‥3),

$$
\begin{aligned}
& -\int_{S} \mathrm{~d} S \mathbf{e}_{\mathrm{t}}^{\mathrm{T}}\left(\partial_{p} \epsilon_{\mathrm{tt}}\right) \nabla_{\mathrm{t}}\left[\epsilon_{z z}^{-1} \nabla_{\mathrm{t}}^{\mathrm{T}} \eta \mathbf{h}_{\mathrm{t}}\right] \\
& =-\int_{S} \mathrm{~d} S \mathbf{e}_{\mathrm{t}}^{\mathrm{T}}\left(\partial_{p} \epsilon_{\mathrm{tt}}\right)\left[-\frac{\omega^{2}}{c^{2}} \eta \mathbf{h}_{\mathrm{t}}+\epsilon_{0} \omega \beta \mathbf{e}_{\mathrm{t}}\right] .
\end{aligned}
$$

Next, using Eqs. (A.7) and (A.9) and integrating by parts the third term on the right-hand side in Eq. (A.3), we find

$$
-\int_{S} \mathrm{~d} S \mathbf{e}_{\mathrm{t}}^{\mathrm{T}} \epsilon_{\mathrm{tt}} \nabla_{\mathrm{t}}\left[\left(\partial_{p} \epsilon_{z z}^{-1}\right) \nabla_{\mathrm{t}}^{\mathrm{T}} \eta \mathbf{h}_{\mathrm{t}}\right]=\epsilon_{0} \omega \beta \int_{S} \mathrm{~d} S\left(\partial_{p} \epsilon_{z z}\right) e_{z}^{2} .
$$

Finally, Eq. (ㅁ) is directly derived using Eqs. (‥10) and (‥11).

\section{APPENDIX B: EFFECTIVE DIELECTRIC TENSOR}

Permittivity is a space-averaged magnitude involving microscopic parameters [28]. At the interface, the averages only change softly, ensuring smooth transitions between media [20]. This description avoids unphysical discontinuities that would otherwise be detrimental for the numerical algorithms [19].

Following $[\underline{19}, 20]$, let us consider an interface between two homogeneous media of permittivities $\epsilon_{1}$ and $\epsilon_{2}$. On the one hand, components of the dielectric tensor affecting the electric field parallel to the interface must be calculated as a weighted average of the permittivities $\langle\varepsilon\rangle=f_{1} \epsilon_{1}+f_{2} \epsilon_{2}$. On the other hand, components of the dielectric tensor acting on the electric field normal to the interface must be computed as the inverse of a weighted average of the inverse permittivities $\langle 1 / \epsilon\rangle=f_{1} / \epsilon_{1}+f_{2} / \epsilon_{2}$. The weight functions $f_{a}$ at each sampling point are evaluated in this work as the relative volume occupied by the $a$ th material in a sphere of radius equal to the sampling distance centered at each sampling point. Finally, the effective dielectric tensor can be written as [19]

$$
\hat{\varepsilon}=\frac{1}{\langle 1 / \varepsilon\rangle} P+\langle\varepsilon\rangle(I-P),
$$

where $I$ is the identity matrix and $P$ is the projection matrix onto the normal to the interface $\hat{\mathbf{n}}$, i.e., $P_{i j}=\hat{n}_{i} \hat{n}_{j}$. It is worth noting that, far from the interfaces, $\hat{\epsilon}$ is a multiple of the identity, recovering the original values for the permittivities $\epsilon_{1}$ or $\epsilon_{2}$.

\section{ACKNOWLEDGMENTS}

This work was financially supported by the Plan Nacional I + D + I under the research project TEC2008-05490, Ministerio de Ciencia e Innovación (Spain), and by the Generalitat Valenciana under the grant PROMETEO 2009-077. Victor Torres acknowledges funding from the Swedish Research Council (VR). D. C.-L. gratefully acknowledges funding from the Generalitat Valenciana (VALi + d predoctoral contract). 
D. C.-L. and E. S. acknowledge programming tasks by Tomás Castelló Rodríguez and Miguel Gea Milvaques.

\section{REFERENCES}

1. N. Ishikura, R. Hosoi, R. Hayakawa, T. Tamanuki, M. Shinkawa, and T. Baba, "Photonic crystal tunable slow light device integrated with multi-heaters," Appl. Phys. Lett. 100, 221110 (2012).

2. M. A. Foster, A. C. Turner, J. E. Sharping, B. S. Schmidt, M. Lipson, and A. L. Gaeta, "Broad-band optical parametric gain on a silicon photonic chip," Nature 441, 960-963 (2006).

3. A. C. Turner-Foster, M. A. Foster, R. Salem, A. L. Gaeta, and M. Lipson, "Frequency conversion over two-thirds of an octave in silicon nanowaveguides," Opt. Express 18, 1904-1908 (2010).

4. X. Liu, B. Kuyken, G. Roelkens, R. Baets, R. M. Osgood, Jr., and W. M. J. Green, "Bridging the mid-infrared-to-telecom gap with silicon nanophotonic spectral translation," Nat. Photonics 6, 667-671 (2012).

5. L. Yin, Q. Lin, and G. P. Agrawal, "Soliton fission and supercontinuum generation in silicon waveguides," Opt. Lett. 32, 391-393 (2007).

6. R. Halir, Y. Okawachi, J. S. Levy, M. A. Foster, M. Lipson, and A. L. Gaeta, "Ultrabroadband supercontinuum generation in a CMOS-compatible platform," Opt. Lett. 37, 1685-1687 (2012).

7. A. Ferrando, E. Silvestre, P. Andrés, J. J. Miret, and M. V. Andrés, "Designing the properties of dispersion-flattened photonic crystal fibers," Opt. Express 9, 687-697 (2001).

8. X. Liu, W. M. J. Green, X. Chen, I.-W. Hsieh, J. I. Dadap, Y. A. Vlasov, and R. M. Osgood, Jr., "Conformal dielectric overlayers for engineering dispersion and effective nonlinearity of silicon nanophotonic wires," Opt. Lett. 33, 2889-2891 (2008).

9. A. C. Turner, C. Manolatou, B. S. Schmidt, M. Lipson, M. A. Foster, J. E. Sharping, and A. L. Gaeta, "Tailored anomalous group-velocity dispersion in silicon channel waveguides," Opt. Express 14, 4357-4362 (2006).

10. S. Mas, J. Caraquitena, J. V. Galán, P. Sanchis, and J. Martí, "Tailoring the dispersion behavior of silicon nanophotonic slot waveguides," Opt. Express 18, 20839-20844 (2010).

11. L. Zhang, Y. Yue, R. G. Beausoleil, and A. E. Willner, "Flattened dispersion in silicon slot waveguides," Opt. Express 18, 20529-20534 (2010).

12. L. Zhang, Q. Lin, Y. Yue, Y. Yan, R. G. Beausoleil, and A. E. Willner, "Silicon waveguide with four zero-dispersion wavelengths and its application in on-chip octave-spanning supercontinuum generation," Opt. Express 20, 1685-1690 (2012).
13. W. H. Press, S. A. Teukolsky, W. T. Vetterling, and B. P. Flannery, Numerical Recipes, 3rd ed. (Cambridge, 2007).

14. S. Jensen and O. Sigmund, "Topology optimization for nanophotonics," Laser Photon. Rev. 5, 308-321 (2011).

15. J. Riishede and O. Sigmund, "Inverse design of dispersion compensating optical fiber using topology optimization," J. Opt. Soc. Am. B 25, 88-97 (2008).

16. E. Silvestre, T. Pinheiro-Ortega, P. Andrés, J. J. Miret, and A. Coves, "Differential toolbox to shape dispersion behavior in photonic crystal fibers," Opt. Lett. 31, 1190-1192 (2006).

17. J. J. Miret, E. Silvestre, and P. Andrés, "Octave-spanning ultraflat supercontinuum with soft-glass photonic crystal fibers," Opt. Express 17, 9197-9203 (2009).

18. S. V. Afshar and T. M. Monro, "A full vectorial model for pulse propagation in emerging waveguides with subwavelength structures part I: Kerr nonlinearity," Opt. Express 17, 2298-2318 (2009).

19. S. G. Johnson and J. D. Joannopoulos, "Block-iterative frequencydomain methods for Maxwell's equations in a plane-wave basis," Opt. Express 8, 173-190 (2001).

20. D. E. Aspnes, "Local-field effects and effective-medium theory: a microscopic perspective," Am. J. Phys. 50, 704-709 (1982)

21. E. Silvestre, T. Pinheiro-Ortega, P. Andrés, J. J. Miret, and A Ortigosa-Blanch, "Analytical evaluation of chromatic dispersion in photonic crystal fibers," Opt. Lett. 30, 453-455 (2005).

22. B. Tatian, "Fitting refractive-index data with the Sellmeier dispersion formula," Appl. Opt. 23, 4477-4485 (1984).

23. L. Zhang, C. Bao, V. Singh, J. Mu, C. Yang, A. M. Agarwal, L. C. Kimerling, and J. Michel, "Generation of two-cycle pulses and octave-spanning frequency combs in a dispersion-flattened micro-resonator," Opt. Lett. 38, 5122-5125 (2013).

24. S. K. Selvaraja, W. Bogaerts, P. Dumon, D. V. Thourhout, and R Baets, "Sub-nanometer linewidth uniformity in silicon nanophotonic waveguide devices using CMOS fabrication technology," IEEE J. Sel. Top. Quantum Electron. 16, 316-324 (2010)

25. R. P. Feynman, "Forces in molecules," Phys. Rev. 56, 340-343 (1939).

26. S. G. Johnson, M. Ibanescu, M. A. Skorobogatiy, O. Weisberg, J. D. Joannopoulos, and Y. Fink, "Perturbation theory for Maxwell's equations with shifting material boundaries," Phys. Rev. E 65, 066611 (2002).

27. E. Silvestre, M. V. Andrés, and P. Andrés, "Biorthonormal-basis method for the vector description of optical-fiber modes," J. Lightwave Technol. 16, 923-928 (1998).

28. J. D. Jackson, Classical Electrodynamics, 3rd ed. (Wiley, 1998). 